\title{
TWO PROPERTIES OF THE FUNCTION $\cos x$
}

HERBERT E. ROBBINS

The function $f(x)=A \cos n(x+B)$, where $A, B$ are any real constants and $n$ is an integer, has the properties:

(I) $f(x)$ is real valued for all real $x$, of period $2 \pi$, and continuous.

(II) $f(x)$ is differentiable, and there exist constants $a, b$ such that, for all $x$,

$$
f^{\prime}(x)=a f(x+b) .
$$

(III) Given any constants $a, a^{\prime}, b, b^{\prime}$, there exist constants $c, d$ such that, for all $x$,

$$
a f\left(x+a^{\prime}\right)+b f\left(x+b^{\prime}\right)=c f(x+d) .
$$

The object of this note is to show that, conversely, any function $f(x)$ which has property (I) and either (II) or (III) is necessarily of the form $f(x)=A \cos n(x+B)$. The latter result is used to derive the parallelogram law of addition of forces from certain other basic assumptions.

THEOREM 1. Let $f(x)$ have properties (I) and (II). Then there exist constants $A, B$ and an integer $n$ such that $f(x)=A \cos n(x+B)$.

Proof. It follows from (II) that $f(x)$ is of class $C^{\infty}$ and hence, from (I), can be represented by a convergent Fourier series, which, moreover, may be differentiated termwise. Thus for some complex constants $k_{n}$,

$$
\begin{gathered}
f(x)=\sum k_{n} e^{i n x}, \quad f^{\prime}(x)=\sum i n k_{n} e^{i n x}, \\
f^{\prime}(x)-a f(x+b)=\sum k_{n}\left(i n-a e^{i n b}\right) e^{i n x} .
\end{gathered}
$$

It follows from (II) that for every integer $n$,

$$
k_{n}\left(i n-a e^{i n b}\right)=0 .
$$

If $f(x) \equiv 0$ then the theorem is trivial. Otherwise, there will exist an $n$ for which $k_{n} \neq 0$. It follows that

$$
\text { in }=a e^{i n b} .
$$

Taking absolute values we have

$$
n= \pm a \text {. }
$$

Thus there can be at most two values of $n$ for which $k_{n} \neq 0$, and these

Received by the editors March 3, 1944. 
values are negatives of one another. Thus for some integer $n$,

$$
f(x)=k_{-n} e^{-i n x}+k_{n} e^{i n x} .
$$

Since, by (I), $f(x)$ is real valued, it follows that $k_{-n}$ and $k_{n}$ are complex conjugates, and the proof is complete.

THEOREM 2. Let $f(x)$ have properties (I) and (III). Then there exist constants $A, B$ and an integer $n$ such that $f(x)=A \cos n(x+B)$.

Proof. Since, by (I), $f(x)$ is continuous and of period $2 \pi$, it possesses at least a formal Fourier series, ${ }^{1}$

$$
f(x) \sim \sum k_{n} e^{i n x}, \quad k_{n}=\frac{1}{2 \pi} \int_{0}^{2 \pi} f(t) e^{-i n t} d t .
$$

By (III), there exist constants $c, d$ such that the function

$$
g(x)=f(x+1)+f(x)-c f(x+d),
$$

continuous and of period $2 \pi$, is identically zero. (This is the only consequence of (III) that we shall use.) Hence

$$
0 \sim \sum k_{n}\left(e^{i n}+1-c e^{i n d}\right) e^{i n x} .
$$

It follows that for every $n$,

$$
k_{n}\left(e^{i n}+1-c e^{i n d}\right)=0 .
$$

If $f(x) \equiv 0$ then the theorem is trivial. Otherwise, there will exist an $n$ for which $k_{n} \neq 0$. For any such $n$ it follows from (9) that

$$
e^{i n}+1=c e^{i n d} \text {. }
$$

Taking absolute values and squaring, it follows that

$$
\cos n=\left(c^{2}-2\right) / 2 \text {. }
$$

Hence if $m$ and $n$ are any two integers for which $k_{m} \cdot k_{n} \neq 0$, it follows from (11) that $\cos m=\cos n$. Hence for some integer $r$,

$$
m= \pm n+2 \pi r \text {. }
$$

Since $\pi$ is irrational, it follows that $r=0$ and $m= \pm n$. Thus the formal Fourier series for $f(x)$ consists of only two terms,

$$
f(x) \sim k_{-n} e^{-i n x}+k_{n} e^{i n x} .
$$

But in this case, since the functions $e^{i n x}$ are complete with respect

1 The proof given here follows a suggestion of Paul R. Halmos. The author's original proof required the unnecessary assumption that $f(x)$ be of class $C^{1}$. 
to continuous functions, the relation $\sim$ can be replaced by an identity,

$$
f(x)=k_{-n} e^{-i n x}+k_{n} e^{i n x} .
$$

Since $f(x)$ is real valued, $k_{-n}$ and $k_{n}$ must be complex conjugates, and the theorem is proved.

We shall now apply Theorem 2 to derive the law of addition of forces. ${ }^{2}$ For simplicity, let us consider only forces acting at a fixed point in a fixed plane in which the angular coordinate $x$ is defined. With such a force we identify the real valued function $F(x)$ which specifies the scalar component of the force in the direction $x$; thus a force is represented by a real valued function of period $2 \pi$. By the sum of two forces $F_{1}(x)$ and $F_{2}(x)$ we mean the function $F_{1}(x)+F_{2}(x)$. Our assumptions are the following.

(i) All forces are geometrically similar. By this we mean that there exists a fixed function $f(x)$ of period $2 \pi$ such that any force $F(x)$ can be written in the form

$$
F(x)=A_{F} \cdot f\left(x+\alpha_{F}\right),
$$

where $A_{F}$ and $\alpha_{F}$ are constants determined by $F(x)$. We need not assume that all values of the constants $A_{F}$ and $\alpha_{F}$ can occur in (15), but we shall assume that there exist at least the forces $F_{1}(x)=f(x)$ and $F_{2}(x)=f(x+1)$.

(ii) The sum of two forces is a force. Together with (i), this implies that the function $f(x)$ has the property that for certain constants $c, d$ and for every $x$,

$$
f(x+1)+f(x)=c f(x+d) .
$$

(iii) The function $f(x)$ is continuous, non-constant, and vanishes for at most two values in the interval $0 \leqq x<2 \pi$.

The proof of Theorem 2 shows that the function $f(x)$, continuous, real valued, of period $2 \pi$, and satisfying (16), must be of the form

$$
f(x)=A \cos n(x+B),
$$

where $n$ is an integer. The hypotheses of (iii) ensure that $n$ can be chosen as 1 . The parallelogram law of addition of forces is an immediate consequence.

United States Naval Academy, Post Graduate School

\footnotetext{
2 See G. Darboux, Bull. Sci. Math. vol. 9 (1875) pp. 281-288; also G. D. Birkhoff,
} Rice Institute Pamphlet vol. 28, no. 1 (1941) pp. 46-50. 